\title{
El valor del informe pericial caligráfico emitido por un laboratorio acreditado
}

The value of the caligraphic expert report issued by an accredited laboratory

Antonio Jesús Llamas Guerra ${ }^{1}$.

https://doi.org/10.5377/rcfh.v6i1.9949

${ }^{1}$ Servicio de Criminalística. Guardia Civil. España

\section{CITAR COMO}

Llamas-Guerra AJ. El valor del informe pericial caligráfico emitido por un laboratorio acreditado. Rev. cienc. forenses Honduras. 2020;6(1): 29-37

\section{ASPECTOS BIOETICOS}

El autor declara que no hubo conflicto de interés en la preparación de este artículo.

\section{RESUMEN}

La mejora constante en el desarrollo y funcionamiento de los laboratorios es una máxima que deben tener todos presentes. En este sentido, al menos en la última década, los laboratorios forenses van implementando en su sistema de gestión las innovaciones que incorpora cualquier otro tipo de empresas u organizaciones como serían los aspectos relativos al personal, a través de normas sobre seguridad e higiene en el trabajo, cuestiones medioambientales dada la preocupación creciente en la sociedad sobre el deterioro medioambiental que se está acrecentando de forma alarmante y que se manifiesta de diversa constante dentro de lo que conocemos como "cambio climático", y por último, la implementación de un sistema de gestión de la calidad, con el fin de exteriorizar un imagen altamente positiva y obtener de esta forma ventajas competitivas sobre otro tipo de empresas que operan en el mismo ramo o sector. Este último aspecto resulta especialmente significativo para los ensayos que, sobre distintas ramas científicas, son realizados por los
Correspondencia a: antoniojesusllamas@hotmail.es

laboratorios forenses, ya que contribuye a fortalecer todo el proceso secuencial analítico seguido y consecuentemente las conclusiones alcanzadas en los análisis realizados.

\section{PALABRAS CLAVE}

Acreditación, Mantenimiento de calidad, Examen de escritura manuscrita, Laboratorio forense.

\section{ABSTRACT}

The constant improvement in the development and operation of the laboratories is a maxim that must be present. In this sense, at least in the last decade, forensic laboratories are incorporating into their management system innovations that incorporate any other type of companies or organizations such as critical aspects of personnel, through standards on occupational health and safety, environmental issues given the growing concern in society about the environmental risk of quality, in order to externalize a highly positive image and obtain this form of competitive advantages over other types of companies operating in the same branch or sector. This last aspect was especially significant for the trials that on different scientific branches are carried out by forensic laboratories, since they contribute to strengthening the entire sequential analytical process followed and consequently the conclusions reached in the analysis performed.

\section{KEYWORDS}

Accreditation; Quality assurance; Handwriting 
examination; Forensic laboratory.

\section{INTRODUCCIÓN}

Como se ha comentado con anterioridad, los laboratorios forenses no se han mantenido al margen de todas aquellas innovaciones o requerimientos que en un momento dado han tenido que adaptar o incorporar, como cualquier organización o empresa, a su estructura organizativa. En este sentido, conviene destacar que durante las últimas décadas se han implementado diversas cuestiones relativas al medio ambiente, a la seguridad e higiene en el trabajo y a la calidad de los productos o servicios ofrecidos por una determinada empresa $u$ organismo.

Son diversas las motivaciones que han generado al final lo que se ha denominado como un "sistema integrado de gestión", que englobaría los aspectos anteriormente comentados, es decir, calidad (normas ISO 9001, 17020 y 17025 Sistema de gestión de calidad), seguridad e higiene en el trabajo (ISO 45001 Sistema de gestión y seguridad en el trabajo) y protección medioambiental (14001 Sistema de gestión medioambiental).

A nivel general podemos considerar que las derivadas de la seguridad e higiene en el trabajo obedece a la aprobación de normativas internas de cada país o en algún caso externas al mismo, como en el supuesto de los países integrados en la Unión Europea, así como la presión y demandas requeridas por las organizaciones sindicales en defensa de los derechos de los trabajadores; en lo referente a cuestiones medioambientales, por la creciente reclamación y concienciación social sobre el deterioro medioambiental acompañado de la aprobación de normativas y acuerdo transnacionales sobre este asunto; y finalmente, la integración de aspectos de calidad derivados del aumento de la competitividad de las empresas y la necesidad de obtener ventajas dentro del mercado en el que se opera, y en algún supuesto por la exigencia impuesta por las Administraciones Públicas de un determinado país o la pertenencia a determinadas Organizaciones nacionales 0 supranacionales.

\section{QUE SIGNIFICA TENER IMPLEMENTADO UN SISTEMA DE GESTIÓN DE CALIDAD Y QUÉ APORTA A UN LABORATORIO.}

La primera pregunta que podemos hacernos cuando escuchamos este término (acreditación) es conocer el verdadero significado del mismo. Atendiendo a la definición más extendida, sería el procedimiento mediante el cual el organismo oficial autorizado, ENAC en el caso de España; aplicando procedimientos de evaluación y verificación independientes, rigurosos y globales (auditorias), reconoce formalmente que un laboratorio (organismo de evaluación de la conformidad) tiene competencia técnica para llevar a cabo tareas específicas (ensayos e inspecciones) conforme a los requisitos recogidos en normas internacionales.

La siguiente cuestión viene determinada por tratar de determinar cuál sería la normativa a aplicar a un laboratorio forense. En este caso, debemos de partir por considerar inicialmente a la norma EN ISO 9001:2015. En dicha norma se establecen los requisitos mínimos relativos a un sistema de gestión de calidad ${ }^{1}$. En concreto, a través del cumplimiento de lo dispuesto en la norma aludida se trata de establecer un proceso de generación de productos o servicios que satisfagan, al mismo tiempo, al cliente, a las leyes y los reglamentos aplicables. Por otra parte, se encuentra la norma EN ISO/IEC 17025:2017, relativa a los requisitos generales para la competencia de los laboratorios de ensayo y calibración ${ }^{2}$. Es esta norma la que afecta más directamente a un laboratorio forense, encuadrándolo dentro de la tipología antes descrita de "ensayo y calibración". En ella se 
contienen los requisitos que permiten a los laboratorios demostrar que operan de forma competente y generan resultados válidos. EI cumplimiento de la normativa anterior conlleva la aceptación y sometimiento, al mismo tiempo, y por norma general, a los criterios de la norma EN ISO 9001:2015. A lo largo del contenido de la norma ISO 17025 se recogen una serie de criterios y requisitos que deben cumplir esos laboratorios, materializados a través del registro y control de cada una de las actividades que realizan con vistas a generar resultados científicamente válidos, lo que resulta más vital si cabe en el seno de un proceso judicial. En resumen, un conjunto de requerimientos que debe cumplir un laboratorio, tras obtener el certificado de acreditación, para mantenerla y que genera sobre el público, entre otras, percepciones favorables de credibilidad del trabajo realizado por esos laboratorios.

Si tuviéramos que situar en una balanza las ventajas y desventajas que conlleva acreditar un laboratorio, claramente las primeras se impondrían sobre las segundas, pudiendo señalar dentro de estas últimas la exigencia de un conjunto de actividades administrativas, complementarias a las existentes, o la existencia de cierta oposición o resistencia por parte del "staff" de la organización donde se trata de implementar.

Dentro de las primeras, es decir, las aportaciones positivas que supone tener un laboratorio acreditado bajo una determinada norma internacional nos encontramos las siguientes:

\section{1.-Asegura los resultados de las pruebas.}

\section{2.-Reconocimiento internacional de los resultados.}

\section{3.-Permite identificar los laboratorios con elevado nivel de calidad.}

\section{4.-Avala los resultados ante clientes $\mathrm{y}$ administraciones.}

\section{5.-Facilita la evaluación contínua del laboratorio.}


Vamos a tratar aclarar algunas de esas características mencionadas.

\section{Asegura los resultados de las pruebas: La} principal razón o motivo por el cual un laboratorio trata de acreditarse es para asegurar que los resultados alcanzados, a través de los distintos ensayos que ejecuta, son correctos y rigurosos fundamentados en la validación de las partes o elementos que intervienen en su elaboración, es decir, los recursos humanos que trabajan en ese laboratorio, los medios materiales o equipamientos empleados en dichos análisis y por supuesto, la aprobación, por parte de la comunidad científica nacional e internacional, del método o protocolo de examen empleado en cada uno de esos ensayos.

Reconocimiento internacional de los resultados: Dicha característica tal vez pasa desapercibida para aquellas personas que trabajan en un laboratorio, que dispone de la correspondiente certificación de acreditación sobre un determinado ensayo.

A nivel general los organismos de acreditación existentes en cada país, en el caso español Entidad Nacional de Acreditación (ENAC), se agrupan en otros regionales (IAAC en América, EA en Europa, etc.). A su vez, estas organizaciones forman parte de una estructura global de acreditación que operan mediante International Laboratory Accreditation Cooperation (I.L.A.C), https://ilac.org/ e International Accreditation Forum (I.A.F), https://www.iaf.nu/.

En las reuniones que organizan dichas entidades se adoptan determinadas decisiones cuya implementación deben seguir las organizaciones regionales y nacionales integradas dentro de su estructura. En este sentido se adoptan, entre otros, Acuerdos de Reconocimiento Mutuo-MRA-, en el caso de I.L.A.C, dentro del campo de la acreditación de los laboratorios y organismos de inspección, que conllevan, entre otras, la aceptación de los resultados alcanzados por esos laboratorios y el reconocimiento mutuo de sus informes, validando de esta forma sus periciales mucho más allá de las fronteras de su país proyectándose a nivel internacional.

\section{Permite identificar los laboratorios con elevado nivel de calidad:}

Como norma general, aquellos laboratorios que tienen acreditados determinados ensayos 0 procesos lo publicitan por la repercusión positiva que tiene para dichas organizaciones. De esta forma aquellas personas o empresas que se dirijan a estos laboratorios para solicitar sus servicios conocen de antemano que el trabajo realizado en esas entidades es riguroso y está sujeto a ciertos controles que se encuentran amparados por esa certificación.

\section{Avala los resultados ante clientes y} administraciones:

Esa certificación que aporta a un laboratorio su acreditación permite disponer de un aval o garantía ante sus clientes, respecto al procedimiento seguido en el análisis demandado y principalmente frente a los resultados derivados de esa actividad.

\section{Evaluación contínua del laboratorio:}

Tal vez este sea uno de los aspectos más importantes a considerar cuando hablamos de la acreditación de un laboratorio. En muchas ocasiones podemos pensar que seguimos un método o protocolo adecuado de trabajo y que contamos con la suficiente formación y experiencia, junto con los medios necesarios, para ejecutar un determinado ensayo sin creer en modo alguno que nuestros resultados puedan ser erróneos frente a todas las consideraciones anteriormente comentadas. 
Es por ello, que la supervisión externa y continúa, llevada a efecto por una entidad como el organismo de acreditación propio de cada país, nos obliga a no desviarnos en modo alguno de las premisas que hayamos adoptado ni confiarnos en modo alguno, conocedores que nuestro trabajo será objeto de evaluación periódicamente.

\section{ACREDITACIÓN DE LOS ENSAYOS SOBRE ANÁLISIS DE TEXTOS MANUSCRITOS O FIRMAS CON FINES IDENTIFICATIVOS.}

Como hemos observado anteriormente la acreditación de determinados ensayos, dentro de un laboratorio, reporta numerosos factores positivos en su evaluación global.

Una de las actividades principales que tienen encomendadas los laboratorios de Documentoscopia o Grafística consiste en la elaboración de informes periciales caligráficos, donde se vierte el resultado del análisis y posterior comparativa de al menos dos textos manuscritos o firmas (dubitadas e indubitadas), en el que se trata de determinar la autenticidad o falsedad del texto y/o firma obrante en el documento cuestionado y/o la posible autoría de dichas muestras, es decir, una actividad que encaja perfectamente con el concepto de ensayo establecido por una entidad de acreditación como E.N.A.C, quien lo define como una operación técnica (realizada por determinados especialistas y con el uso de equipos específicos) que consiste en determinar una o más características de un material (en nuestro caso, el examen de un conjunto de parámetros asociados a una escritura o firma con la finalidad de determinar si fue realizada o no por la persona a cuya identidad se atribuye) de conformidad con un procedimiento (protocolo de examen de escritura y firmas seguido por un laboratorio de Documentoscopía). Aunque estamos hablando de una disciplina forense cuya investigación científica se ha producido a lo largo de una dilatada historia, que abarca varios siglos y que se ha plasmado mediante la fortaleza de los métodos aplicados en su análisis, debemos de ser conscientes que el factor humano, es decir, el perito calígrafo, es pieza fundamental en ese tipo de examen, a pesar de disponer de la ayuda inestimable de cierto instrumental científico. Su formación y experiencia son básicas para realizar un adecuado examen y alcanzar unos resultados plenamente satisfactorios que se materialicen, por medio de unas certeras conclusiones, en sus informes periciales. En el mismo sentido se pronuncian Dilek Salkim Islek y Emel Hulya, en relación con el artículo realizado por ésos sobre la acreditación, bajo la norma EN ISO/IEC 17025:2017, de los laboratorios forenses turcos ${ }^{3}$. Sin entrar en detalles sobre un tipo específico de laboratorio señalan que la competencia del personal es fundamental. Según citan es uno de los capítulos donde se centra principalmente la norma comentada (EN ISO/IEC 17025:2017), indicando a través de ella una serie de requisitos que deben cumplir los laboratorios que pretendan acreditarse, en particular, la necesidad de evaluar y registrar sus conocimientos y habilidades, estado de educación y necesidades de capacitación. En base a lo anterior, queda suficientemente palpable que el componente humano juega un papel decisivo en este tipo de exámenes. La implementación de un sistema de gestión de la calidad por parte de los laboratorios forenses, junto a la propia acreditación del ensayo antes comentado sobre el análisis de textos manuscritos y/o firmas con fines identificativos, aportan a dichos laboratorios un alto grado de fortaleza y seguridad a la hora de afrontar ese tipo de análisis, al cumplir sus especialistas, entre otros requerimientos, con una adecuada formación y estar sometidos a un proceso de evaluación continua, mediante la ejecución de periódicos 
controles internos y externos que garantizan la cualificación de dicho personal, estableciéndose al efecto procedimientos que evalúan sus resultados y marcan ciertas directrices de actuación para aquellos supuestos en los que se detecten ciertos errores.

A pesar de lo anterior, existen muchos países donde el examen pericial caligráfico no se encuentra regulado dentro de su sistema educativo general, como sería el caso español. Basta realizar determinadas actividades formativas, de duración y credibilidad no suficientemente contrastada, para poder ejercitar la actividad de "perito calígrafo". Las periciales realizadas por ésos son las únicas opiniones vertidas por expertos con las que cuentan en ocasiones las Autoridades Judiciales encargadas del enjuiciamiento de un determinado procedimiento judicial para sustentar, junto con otros elementos probatorios, su decisión a través de la sentencia correspondiente. En otros supuestos, esas mismas periciales son enfrentadas en un procedimiento judicial junto a otras elaboradas por un laboratorio acreditado, pudiendo encontrarnos ante situaciones en las que las conclusiones de ambos sean coincidentes o no. En cualquier de las dos situaciones comentadas, ciñéndonos a la situación de España, la Autoridad Judicial competente en dicho procedimiento, en base a lo establecido la Ley de Enjuiciamiento Criminal, ese tipo de pruebas será objeto de apreciación libre por el Tribunal, establecimiento como límite a dicha valoración las reglas de la sana crítica, debiendo entenderse ésta como las más elementales directrices de la lógica humana, apreciándolas junto al resto de actividad probatoria practicada en el proceso como se señala en la Sentencia del Tribunal Supremo de España $124 / 17$, de fecha $24.02 .2017^{4}$, por lo que podemos encontrarnos ante casos en los que se valore, en mayor medida, alguna de las dos periciales o no se tengan en consideración ninguna de ésas (generalmente ocurre este último supuesto cuando las conclusiones de las periciales aportadas son totalmente discrepantes entre sí).

A través de lo expuesto, no queremos en modo alguno indicar que las conclusiones realizadas por un laboratorio carente de acreditación, en ese ensayo, sean erróneas o presenten menor credibilidad que uno acreditado, simplemente lo que pretendemos es dejar suficientemente claro qué supone implementar un sistema de gestión de calidad en un laboratorio y tener acreditado cierto tipo de ensayos, como el comentado.

Sobre este particular, resulta interesante el estudio realizado por Mauricio Duce, Profesor de Derecho, sobre el uso y prácticas de la prueba pericial en el proceso penal chileno ${ }^{5}$. A lo largo de dicho estudio, pone de manifiesto una serie de deficiencias, según su punto de vista, sobre la apreciación, por parte de las Autoridades Judiciales, de las pruebas periciales presentadas, entre otros, por los distintos laboratorios forenses oficiales de dicho país. Tras su análisis, establece la existencia de una serie de causas que a su juicio suponen a la postre que dichos informes periciales tengan "mayor peso en la práctica y menor capacidad de control de su calidad por parte de los abogados y actores del sistema legal", y que, de esa forma, puedan generar un cierto riesgo si se valora excesivamente una prueba pericial "poco confiable, si los peritos prestan testimonio inválido, o si se comportan derechamente mal". A la vista de esas consideraciones, a la hora de evaluar en qué tipo de laboratorios o sobre qué ensayos existe una percepción más positiva sobre la fortaleza de la calidad de los peritajes obtuvo de su estudio que esa mayor garantía pericial, venía ligada a nivel internacional, con el establecimiento de sistemas de acreditación de laboratorios forenses, los cuales permiten verificar si 
efectivamente los procesos de trabajo en los mismos responden a estándares de calidad, lo que asegura la confiabilidad de sus resultados, haciendo especial mención sobre la disponibilidad o no de acreditación bajo el amparo de la norma ISO 17025 de determinados laboratorios forenses. Apoyando lo comentado por Mauricio Duce en su artículo, centrando en nuestro caso la atención sobre la apreciación, por parte de las Autoridades Judiciales, de las pruebas periciales caligráficas, podemos señalar que, aunque no existen estudios en España que avalen o certifiquen la existencia de una percepción más positiva de ese tipo de periciales, emitidas por laboratorios acreditados, se va constatando como ésas van adquiriendo progresivamente mayor peso, en un mismo procedimiento judicial, frente a otras emitidas por laboratorios que no tienen acreditado dicho ensayo, al generar en las Autoridades Judiciales una mayor confianza, dados los resultados contradictorios que, en numerosas ocasiones, se producen en las conclusiones vertidas en ese tipo de periciales. Ejemplo de lo anterior lo encontramos en la Sentencia número 732/14, dictada por la Audiencia Provincial de Madrid, con fecha dos de octubre de dos mil catorce, en relación con unos presuntos delitos de estafa y falsificación de documento mercantil, en cuyo procedimiento se aportaron, entre otros, dos informes periciales caligráficos, uno de ellos realizado por el Laboratorio de Grafística de la Guardia Civil, acreditado en el ensayo de análisis de textos manuscritos y firmas en caracteres latinos bajo la norma ISO/IEC 17025. El Órgano Judicial sentenciador estimó en mayor medida el informe pericial de Guardia Civil, al señalar textualmente que "se trata de un dictamen extenso y detallado, generoso en fotografías, explicaciones, cotejos y detalles, avalado en buena parte por la acreditación de ENAC, lo que no es predicable de los otros informes" 6 .

Resulta evidente, en el supuesto español, que la acreditación de los ensayos sobre análisis de textos o firmas manuscritas, aporta una serie de hechos favorables en la defensa de los informes elaborados al efecto en las vistas orales. Buena parte de las preguntas que se formulan a esos especialistas, centradas mayoritariamente en su cualificación y/o experiencia y en menor medida, en el protocolo de examen seguido, quedan perfectamente respondidas mediante la indicación de tener acreditado el ensayo y todo lo que ello conlleva.

A nivel general, suscita menor posibilidad de cuestionar el examen y las conclusiones alcanzadas, si lo comparamos con otro tipo de ensayos similares realizados por laboratorios no acreditados. Como se ha explicado al comienzo del presente, la acreditación otorgada por la entidad correspondiente determina la competencia técnica que tiene un laboratorio concreto para llevar a cabo tareas específicas (ensayos e inspecciones) conforme a los requisitos recogidos en normas internacionales, y de esta forma, en el caso español, se fortalece esa carencia de una formación oficial reglada sobre el examen pericial caligráfico de textos manuscritos y firmas y/o sobre la exigencia de una determinada experiencia en ese terreno.

Aunque el foco de atención de este artículo se ha centrado sobre el componente humano, es decir, el especialista involucrado en la ejecución del correspondiente análisis de textos manuscritos o firmas con fines identificativos, eso no quiere decir que sea la única parte que interviene en ese examen, por el contrario, como cualquier otro tipo de ensayo de las distintas disciplinas forenses que existen, en el caso particular de la pericia caligráfica encontramos otros componentes que deben tenerse en consideración como son los distintos equipos e instrumental empleado, la disponibilidad de adecuadas instalaciones o el propio método de ensayo seguido en dicho análisis, entre otros. 
Como bien sabemos, cualquier laboratorio que pretenda acreditarse en un determinado ensayo debe someterse al cumplimiento de una serie de requisitos en varios aspectos. En el caso particular de un laboratorio de Documentoscopia, los equipos necesarios disponibles en el mismo como video espectro comparadores, macroscopios, etc., requieren disponer de un plan de mantenimiento y calibración para asegurar en todo momento un funcionamiento correcto y una exactitud en sus mediciones; las instalaciones así como las condiciones ambientales deben ser adecuadas para esas actividades propias del laboratorio y no afectar adversamente a la validez de los resultados de esos ensayos practicados; y en el caso de los métodos o procedimientos empleados, ésos deben apropiados para la actividad pericial caligráfica, garantizándose mediante la oportuna validación de dicho método por la comunidad científica. En resumen, el cumplimiento de una serie de requisitos y el seguimiento y control sobre cada una de esas partes que de una u otra forma pueda influir en sus resultados.

Una vez decidida la necesidad de acreditar un determinado ensayo como el comentado deberíamos de plantearnos cuál sería la norma ISO aplicable. Sobre este particular la discusión se centra básicamente en dos normas: la ISO/IEC 17020 o la ISO/IEC 17025, la primera, más centrada en entidades de inspección, y la segunda, en los laboratorios de ensayo y calibración. En el caso del Laboratorio de Grafística integrado dentro de la estructura del Servicio de Criminalística de la Guardia Civil de España, llegado el momento de afrontar el proceso de acreditación en determinados ensayos, en torno al año 2009, se orientó ése hacia la norma ISO/IEC 17025, no sólo por considerar que era la que se ajustaba al trabajo que se pretendía acreditar, sino porque así también lo entendió la entidad oficial española de acreditación E.N.A.C. Esa postura quedó igualmente reforzada por la propia Red Europea de Laboratorios de Ciencias Forenses (ENFSI), quien dentro de los requisitos establecidos por dicho Organismo para pertenecer a él, como es el caso del laboratorio de la Guardia Civil de España, establecía la necesidad de haberse acreditado o realizar las pasos necesarios para su consecución en los distintas actividades de pruebas de laboratorio según la norma ISO/IEC 17025, dejando la norma ISO/IEC 17020 para aquellas otras actividades más propias de inspección como el trabajo efectuado en la escena del crimen.

\section{CONCLUSIÓN}

Una de las mejoras que vienen realizando los laboratorios forenses en los últimos años viene determinada por la implementación de un sistema de gestión de calidad en el mismo acompañado por la acreditación de cierto tipo de ensayos o análisis.

Como ha quedado expuesto no debe valorarse la acreditación de un informe pericial caligráfico desde la perspectiva de su mayor o menor consideración por parte de las Autoridades Judiciales, donde al menos en el caso español se deja a ésas bajo su libre apreciación frente a otros ensayos similares no acreditados, sino desde el prisma de considerar los innumerables elementos positivos que reporta dicha acreditación y que se translucen en la generación de una mayor fortaleza y confianza no sólo por parte de los potenciales clientes que puedan demandar sus servicios sino por el público en general.

\section{REFERENCIAS BIBLIOGRÁFICAS}

1.- Organización Internacional de Estandarización Norma ISO/EC 9001. 5aed. Ginebra: ISO; 2015.

2.- Organización Internacional de Estandarización. Norma ISO/EC 17025. 3aed-. Ginebra: ISO; 2017.

3.-Islek DS, Yukseloglu EH. Accreditation of forensic science laboratories in Turkey in the scope of TS EN 
ISO/IEC 17025:2017 standard. Med Sci.

Disponible

en:

[Internet].2018[consultado 12 de marzo https://scielo.conicyt.cl/scielo.php?script=sci artte 2020];7(4):962-6.

Disponible

en

http://www.medicinescience.org/review-articleaccreditation-of-forensic-science-laboratories-inturkey-in-the-scope-of-ts-en-iso-iec-170252017standard/

4.-Tribunal Supremo, Sala Primera de lo Civil (Es) Sentencia número 124/17 (STS 124/2017), 24 de febrero de 2017. [Internet]. España: Vlex; 2017. [consultado 12 de marzo 2020] Disponible en: https://supremo.vlex.es/vid/671290581.

\section{xt\&pid=S0718-33992018000100042}

6.-Sentencia de la Audiencia Provincial de Madrid de España (Sección 30) 732/14, de fecha 02.10.2014, dimanante del Procedimiento Origen 632/04 del Juzgado de Instrucción num.6 de Alcalá de Henares (Madrid). Disponible en https://www.iberley.es/jurisprudencia/sentenciapenal-n-732-2014-ap-madrid-sec-30-rec-83-201202-10-2014-1494179

5.-Duce M. Una aproximación empírica al uso y prácticas de la prueba pericial en el proceso penal chileno a la luz de su impacto en los errores del sistema. Polít crim.[ Internet] 2018[consultado 12 de marzo 2020];13(25):42-103. 